\title{
Cushing's Syndrome
}

National Institute of Neurological Disorders and Stroke (NINDS)

\section{Source}

National Institute of Neurological Disorders and Stroke (NINDS). Cushing's Syndrome

Information Page.

Cushing's syndrome, also called hypercortisolism, is a rare endocrine disorder caused by chronic exposure of the body's tissues to excess levels of cortisol - a hormone naturally produced by the adrenal gland. Exposure to too much cortisol can occur from long-term use of synthetic glucocorticoid hormones to treat inflammatory illnesses. Pituitary adenomas (benign tumors of the pituitary gland) that secrete increased amounts of ACT H (adrenocorticotropic hormone, a substance that controls the release of cortisol) can also spur overproduction of cortisol. Tumors of the adrenal gland and ectopic ACTH syndrome (a condition in which ACTH is produced by various types of potentially malignant tumors that occur in different parts of the body) can cause similar problems with cortisol balance. Common symptoms of Cushing's syndrome include upper body obesity, severe fatigue and muscle weakness, high blood pressure, backache, elevated blood sugar, easy bruising, and bluish-red stretch marks on the skin. In women, there may be increased growth of facial and body hair, and menstrual periods may become irregular or stop completely. Neurological symptoms include difficulties with memory and neuromuscular disorders. 